\title{
Educación para la \\ salud, tarea \\ compartida
}

UT. Revista de Ciències de l'Educació

Juny 2011. Pag. 25-40

ISSN 1135-1438

http://pedagogia.fcep.urv.cat/revistaut

\author{
Carlos Rosales López ${ }^{\mathrm{a}}$ \\ Rebut: 18/03/2011 Acceptat: 06/05/2011
}

\section{Resumen}

En este trabajo se reflexiona sobre el concepto actual de salud y de educación para la salud y se analizan las características de los diversos agentes que intervienen en la misma, de manera específica, la familia, la escuela y la comunidad local. Se toma como referencia la situación actual de la educación para la salud en España, tras la evolución experimentada desde el final de la dictadura.

Se parte del supuesto de que la actuación de dichos agentes no puede entenderse de forma independiente sino estrechamente relacionada y se ponen de relieve ámbitos destacados de colaboración.

Palabras clave: Educación para la salud, familia, escuela, comunidad.

\section{Health education, shared responsability}

\section{Abstract}

In this paper I reflect on the present concept of health and health education and analyse the characteristics of differents agents that contribute to it: family, school and community. It draws on the current status of health education in Spain following the developments since the end of dictatorship.

It starts with the assumption that the intervention of this agents aren't independent but closely related and show significant fields of collaboration.

Key words: Health education, family, school, community

\section{Concepto de Salud}

El cuidado y la mejora de la salud, la superación de enfermedades, ha constituido una aspiración constante del ser humano a lo largo del tiempo. En todas las culturas es 
posible identificar rasgos de esta preocupación. Así, en Grecia conviven la explicación mitológica con el ejercicio de racionalización. En Roma se acentúa la preocupación por la salud pública. En la cultura árabe se realizan grandes progresos teóricos, técnicos y prácticos. En la Edad Media se refuerza la vinculación de los estados de salud y enfermedad con las creencias religiosas. En el Renacimiento se fortalece la investigación, que desde entonces hasta la actualidad no deja de realizar grandes avances. En el siglo XIX tiene su origen la investigación psicológica, que en lo sucesivo se combinará con la de tradición naturalista y aportará valiosos instrumentos de carácter psicoterapéutico.

La asistencia sanitaria, paralela al desarrollo del conocimiento sobre la salud ha corrido a cargo en la Antigüedad y la Edad Media de instituciones filantrópicas, religiosas, vinculadas a la monarquía y a la nobleza o a los gremios de trabajadores. A partir del Renacimiento y con la conformación de las nuevas nacionalidades serán las administraciones de cada país quienes se harán cargo de los servicios de salud. Ya en el siglo XX se crean grandes organismos de carácter internacional coordinadores e impulsores de la asistencia, la investigación y la educación de las gentes en el terreno de la salud.

La Organización de las Naciones Unidas (ONU), creada en 1945 considera la salud como un derecho humano fundamental a partir de la Asamblea General celebrada en París en 1948, en la que tuvo lugar la Proclamación Universal de los Derechos Humanos. Y la Organización Mundial de la Salud (OMS), como organismo de la ONU, ha proyectado grandes esfuerzos en la promoción de la salud a nivel mundial. A ella se deben las más conocidas y aceptadas definiciones de salud como estado óptimo de bienestar físico, mental y social y de educación para la salud como capacitación de las personas y las comunidades para ejercer control sobre los factores que afectan a la salud.

El análisis de la evolución histórica del concepto de salud pone de relieve la existencia en el mismo de una enorme serie de factores intensamente interactuantes. Así, existe una salud individual, propia de cada persona, con características diferentes a la de los demás. Existe al tiempo, una salud comunitaria o grupal, configurada por rasgos comunes a poblaciones de distinta extensión. Existe una salud de carácter subjetivo, configurada por las percepciones que cada persona tiene sobre sí misma, sobre su bienestar o malestar, por sus vivencias de salud y enfermedad. Al tiempo se puede entender que existe una salud objetivable, reflejada a través de indicadores constatables.

Se puede entender la salud de forma diacrónica si realizamos un seguimiento temporal de la misma a lo largo de la vida de una persona o comunidad. Y también se puede analizar la salud de forma sincrónica, en un determinado momento.

La salud puede entenderse en sentido restringido como carencia de enfermedad o en sentido ampliado como prevención y promoción del bienestar. Y este bienestar, como se indica en la definición propuesta por la OMS debe entenderse en los planos de lo físico, lo mental y lo social. 
El estudio de una realidad tan compleja desborda los límites de una disciplina, de modo que en la actualidad debe considerársela desde una perspectiva multidisciplinar. A las ciencias de la salud, tradicionalmente vinculadas a la medicina se van agregando en estos momentos otras como la psicología (estudio de componentes mentales, desarrollo de la psicoterapia), las ciencias ambientales (influencia del medio ambiente y sus alteraciones en los estados de salud y enfermedad), la sociología (influencia del entorno social como la familia y la comunidad local), la legislación y administración (prestación de servicios, normativas laborales, etc), las ciencias de la educación (capacitación a personas y comunidades para la prevención y promoción de la salud, para el enfrentamiento a problemas o enfermedades...

\section{Educación para la salud}

Podría darse el caso de personas que con una dotación genética óptima y residiendo en entornos no agresivos, contaran asimismo con buenos servicios sanitarios y sin embargo no gozaran de buena salud porque sus estilos de vida (organización de periodos de actividad y descanso, alimentación, forma de conducir, consumo de sustancias nocivas, clase de entretenimientos...), no fueran saludables.

La intervención educativa encuentra su fundamento en la necesidad de fomentar un adecuado conocimiento sobre la salud y la enfermedad, así como de desarrollar hábitos y actitudes correspondientes a formas de vida saludable. La educación se proyecta pues en la estimulación del pensamiento, los sentimientos y las actuaciones de las personas.

Es muy posible que la educación para la salud tenga unos antecedentes tan remotos como los de la asistencia sanitaria. Desde antiguo los profesionales de la salud no se han limitado a curar sino que se han caracterizado por proporcionar importantes consejos a sus pacientes para evitar la enfermedad o lograr mejores estados de bienestar. En este sentido, los profesionales de la salud (médicos, enfermeras, fisioterapeutas...) han actuado y en la actualidad lo siguen haciendo como eficaces educadores.

Ahora bien, es la familia el ámbito de vida en el que se realizan los primeros y quizás fundamentales aprendizajes sobre la salud, por sus importantes repercusiones a lo largo del tiempo y por la predisposición que crean en la persona a realizar otros aprendizajes con posterioridad en la misma línea de los primeros.

La escuela es otro gran escenario de la educación para la salud. En ella discurren bastantes años de la vida y se realizan aprendizajes que se coordinan con los iniciales de carácter familiar, incidiendo predominantemente en los terrenos del conocimiento y la comprensión.

El lugar de trabajo, otro relevante contexto de vida de la persona, debe constituirse asimismo en un entorno estimulante de la salud en términos generales y de manera 
más intensa en aquellos aspectos en los que existen riesgos específicos de la profesión.

La comunidad local constituye cada vez más un agente de salud mediante el apoyo a las personas a través de servicios diversos: tiempo libre, asistencia sanitaria, rehabilitación, apoyo a la familia, etc.

En definitiva, todos aquellos ámbitos en los que transcurre la vida de las personas están llamados a desempeñar una función educadora y esta tarea no debe entenderse como un conjunto de actuaciones puntuales y diferenciadas, sino como intervenciones estrechamente coordinadas.

\section{Intervención familiar}

La influencia educativa de la familia se ejerce a través de la comunicación y la vivencia de modelos que inducen estilos de vida. Esta influencia se produce desde los primeros momentos de la vida y tiende a permanecer y a promover otros aprendizajes en el futuro en relación con los realizados con anterioridad.

Es cierto que desde una perspectiva actual podemos pensar que la eficacia de la influencia familiar ha descendido respecto a la que se ejercía en otros tiempos ya que en muchas ocasiones la familia se reduce a los padres y un hijo o dos, con lo que no existe relación del niño con otras personas, especialmente los abuelos. Por otra parte, el hecho de trabajar en muchas ocasiones la madre, determina que el niño se escolarice antes y que el periodo de vida familiar en el hogar se limite considerablemente, con lo que los dos grandes instrumentos educativos del diálogo y el ejemplo, se reducen considerablemente. Hay que subrayar además que en estos momentos los niños permanecen ante las pantallas de televisión y ordenador una elevada cantidad de tiempo.

No obstante, la importancia cualitativa de la influencia familiar sigue siendo muy elevada y se manifiesta en múltiples dimensiones de la salud. Por ejemplo:

a) La prevención de enfermedades contagiosas clásicas y de accidentes, a través de la higiene personal y del conocimiento y cuidado en la manipulación de objetos, fármacos $u$ otros productos peligrosos.

b) La prevención de enfermedades mentales mediante la creación de un clima estimulante de la autoestima y de relaciones personales gratificantes.

c) La educación afectiva y sexual, mediante la creación de un clima de confianza en el que el niño se atreva a dialogar y plantear preguntas sobre cuestiones que desconoce y le preocupan.

d) Alimentación. El conocimiento de las características de la alimentación y de las repercusiones que puede tener en la salud, son aprendizajes que el niño puede realizar en el hogar a través como en casos anteriores del diálogo 
con sus padres, de los modelos que éstos presentan y de su participación en las tareas cotidianas.

e) Drogas. La familia puede contribuir a la tarea de prevención del consumo de drogas a través de la información y la creación de un clima familiar alejado del consumo de drogas como el alcohol y el tabaco.

f) Educación vial. Como en otros temas la actuación de los padres presenta un importante valor modélico tanto en el comportamiento como peatón como en la utilización de vehículos

g) Uso de medios de comunicación social, redes informáticas, videojuegos. A partir de un análisis crítico de las características de los programas de estos medios se pone de relieve la necesidad de que los padres asuman su responsabilidad en torno a la regulación del tiempo de uso de los medios y la selección y crítica de los programas que siguen sus hijos.

\section{La escuela, agente de educación para la salud}

La escuela constituye un destacado escenario de actividad y vida de la persona durante un periodo prolongado de tiempo. En nuestro país la escolaridad obligatoria abarca diez años (de los seis a los dieciséis en las etapas de educación primaria y secundaria obligatoria) y cada vez es más numerosa la población infantil que se escolariza varios años antes (de tres a seis años), así como la que prolonga su estancia en centros educativos más allá de los dieciséis (repeticiones de curso, bachillerato, formación profesional...)

En la medida en que a través de la escolaridad obligatoria se pretende estimular el desarrollo completo del alumno mediante una atención educativa de calidad, la salud constituye un tema de obligado tratamiento en los centros escolares, ya que no es imaginable sin ella cualquier otra forma de perfeccionamiento y enriquecimiento personal y social.

La escuela deberá convertirse en un ambiente saludable en sus dimensiones materiales, organizativas y funcionales. En este sentido, debemos pensar en centros con unas determinadas características en sus instalaciones, espacios, insonorización, temperatura, entornos libres de contaminación, posibilidades de circulación fácil, instalaciones sanitarias y de otro tipo adaptadas a la integración de alumnos con necesidades educativas especiales, etc.

Los muebles y materiales didácticos deberán poseer unas características ergonómicas, tamaño, manejabilidad, carencia de riesgos en su utilización, etc., de acuerdo con el nivel madurativo de los alumnos.

La organización de actividades, su distribución en el horario se ha de acomodar a las posibilidades de trabajo y a los indicadores de fatigabilidad del alumno. 
Desde una perspectiva organizativa y funcional, el desarrollo de la educación para la salud implica se tomen en consideración a nivel de proyecto educativo una amplia serie de condicionantes como los siguientes:

- El estudio de las características socioculturales de la población y en concreto de los alumnos, con especial atención a sus características de salud y enfermedad.

- La asunción, a nivel de señas de identidad y de objetivos generales del centro, de una clara aspiración a la mejora de la salud de los alumnos.

- La promoción entre los profesores y demás profesionales del colegio de una actitud claramente positiva en torno a dichos objetivos y la realización de actividades de actualización y perfeccionamiento profesional en relación a su enseñanza.

- El establecimiento de vínculos de colaboración con otras instituciones pertenecientes a la comunidad que puedan contribuir a la realización de actividades de educación para la salud fuera o dentro del centro, tomando especialmente en consideración a los padres y madres de alumnos y a sus asociaciones.

A nivel de proyecto curricular, la implantación de la educación para la salud sigue la misma línea que otros temas transversales, es decir, se la considera como un conjunto de aprendizajes que sin estar recogidos en un área determinada se han de enseñar de forma implícita, a través de todas las materias convencionales y constituyendo como una modificación cualitativa de las mismas. En este sentido, se entiende que al enseñar temas de lengua, de matemáticas, de conocimiento del medio o de cualquier otra materia convencional, se tratarán al tiempo en todas ellas cuestiones vinculadas con la salud y la enfermedad. Esta consideración transversal no impide que en determinados momentos se desarrollen temas específicos sobre la salud mediante unidades didácticas centradas en ellos o que se realicen actividades a nivel global del centro (Conmemoraciones, semanas de...).

En la elaboración del proyecto curricular, el profesorado, organizado en equipos o departamentos ha de realizar una cuidadosa programación vertical y horizontal de objetivos y contenidos en educación para la salud tomando en consideración tanto el interés de los temas en función del nivel madurativo de los alumnos como las posibilidades de tratamiento e cada área curricular o asignatura, así como las posibilidades de colaboración interdisciplinar entre ellas. La comisión de coordinación pedagógica de los centros puede desempeñar un papel fundamental en la realización de una programación adaptada y coherente.

De todos modos, el carácter transversal de la salud posibilita el que en todo momento, cualquier ocasión pueda utilizarse como estímulo para el desarrollo de estas cuestiones sin límites de asignaturas o de horarios. 
La educación para la salud en la escuela deberá presentar una doble perspectiva: preventiva y curativa. En el primer caso mediante la información, la sensibilización, la promoción de hábitos y actitudes que alejen al alumno de peligros para la salud. En el terreno curativo, mediante una intervención de apoyo a alumnos que padezcan alguna enfermedad (diabetes, sida, anorexia...). Este apoyo puede entenderse en el plano propio de la salud en coordinación con la familia (por ejemplo, para el seguimiento de tratamientos), y en el plano académico o de aprendizaje escolar. En este último sentido, el departamento de orientación del centro tiene la responsabilidad de realizar un cuidadoso diagnóstico de las dificultades de aprendizaje, con frecuencia asociadas a periodos de ausencia del centro, y a partir del mismo, elaborar las medidas de refuerzo y en su caso de adaptación curricular que sean necesarias.

\section{Intervención de la comunidad en educación para la salud}

Las formas de intervención de la comunidad social en educación para la salud pueden analizarse en distintos niveles: internacional, nacional y autonómico o local.

Se destacan en el plano internacional las iniciativas de la ONU y de diversas organizaciones nacidas en el seno de la misma. Así, la ONU reconoce como un derecho humano fundamental el de la salud en los artículos 1, 3 y 25 de la Proclamación Universal de Derechos Humanos.

Dentro de la ONU se creó la OMS (Organización Mundial de la Salud), que desde su origen ha venido impulsando la investigación, la educación para la salud y la puesta en marcha de planes, programas y proyectos para la mejora de las condiciones de salud de la población en cualquier país del mundo y especialmente en los menos desarrollados. Por iniciativa de esta organización se han celebrado diversas conferencias internacionales sobre prevención y promoción de la salud, en las que se hace referencia a la educación como un agente relevante.

La Organización de las Naciones Unidas para la Educación, la Ciencia y la Cultura (UNESCO), creada también en la década de los cuarenta, desarrolló el Proyecto de Escuelas Asociadas, actualmente más de siete mil y los Clubes de Amigos y de Universidades Asociadas. Todas estas instituciones se han comprometido con la educación de las personas en el respeto a los derechos humanos fundamentales y la elevación del nivel de vida, especialmente en los sectores de población más deprimida.

El Fondo de las Naciones Unidas para la Infancia (UNICEF) se creó dentro de las Naciones Unidas tras la segunda guerra mundial con el objetivo básico de atender a los numerosos niños que se encontraban en estado de gran precariedad física, afectiva y social a consecuencia de las calamidades padecidas. Una vez cumplido este objetivo, la UNICEF extendió su ámbito de intervención a la infancia que en todo el mundo se encuentra en situación deficitaria, de riesgo, abandono, abuso, etc. 
El Programa de las Naciones Unidas sobre el SIDA (ONUSIDA) fue creado por iniciativa de la OMS y en él colaboran diversos organismos e instituciones como la UNESCO, el UNICEF, el Banco Mundial, la OIT y diversas ONGS. Con sede en Ginebra, los objetivos del programa son prevenir la extensión de la enfermedad y prestar apoyo a quienes la padecen. Se realizan actividades de información y educación así como de atención sanitaria y suministro de medicamentos. Para el siglo XXI se fija como objetivo reducir a la mitad la cantidad de enfermos que la padecían en el 2000.

\section{Comunidad nacional y autonómica}

Los países que forman parte de organizaciones como las citadas, aplican en su propio ámbito las normativas que les afectan y además elaboran y aplican su propia legislación, relacionada con la de carácter internacional. Así, las directrices de la ONU, UNESCO, UNICEF, OMS, FAO, etc., se reflejan en nuestras principales leyes estatales y autonómicas, comenzando por la Constitución de 1978. De manera específica, a nivel estatal se pueden citar las siguientes:

LOGSE (Ley de Organización General del Sistema Educativo, 1990). A partir de esta ley se introducen en los proyectos educativo y curricular de los centros de enseñanza los denominados "contenidos transversales", cuestiones de gran interés social y personal como la convivencia, la conservación del medio ambiente, la igualdad hombre-mujer o la salud. Estos temas no se organizan en asignaturas específicas sino que se tratarán "a través de" las áreas y materias convencionales, constituyendo como una modificación cualitativa de las mismas.

LOE (Ley Orgánica de Educación, 2006). Se trata de una ley que enlaza con la anterior, constituyendo en muchos aspectos una continuación e intensificación de la misma. En ella se destaca la importancia de la educación en valores como temas transversales y se crea una disciplina denominada "Educación para la ciudadanía", que abarcará aprendizajes relativos derechos y deberes de la persona como ciudadano, entre ellos los correspondientes a la salud en sus dimensiones personal y comunitaria.

Ley General de Sanidad (1986). El ella se organiza el sistema de atención sanitaria en nuestro país. En diferentes títulos de la misma se hace referencia a la promoción de la salud y a la prevención de enfermedades como importantes objetivos del sistema sanitario. Especialmente en los títulos primero, tercero y quinto se mencionan la información y la educación sanitaria, considerándolas fundamentales para mejorar la salud individual y comunitaria.

Ley de Medidas Sanitarias frente al Tabaquismo (2005). En ella se pone de relieve el hecho de que el consumo de tabaco constituye en nuestro país como en otros de nuestro entorno la primera causa de mortalidad y morbilidad y se deduce la necesidad de adoptar medidas para evitarlo o reducirlo. 
De manera específica, se trata de establecer espacios sin humo, especialmente para menores, se prohíbe la publicidad del tabaco así como su venta a menores de dieciocho años. En el capítulo IV de esta ley se contienen disposiciones relativas a la información y la educación.

Esta Ley aparece modificada por la Ley 42/2010 de 30 de diciembre, en la que se intensifican las medidas protectoras de la salud respecto a los peligros del consumo de tabaco.

Plan Nacional Sobre Drogas (PNSD). Surge por iniciativa del Ministerio del Interior en 1985 para coordinar y potenciar diversas actuaciones de administraciones públicas y entidades sociales en torno a la prevención del consumo de drogas y la deshabituación y reinserción social de los consumidores.

Comisión Interministerial de Circulación Vial. Se creó en 1979 y se remodeló en 2005. Está constituida por diversos ministerios y pretende la coordinación de esfuerzos para reducir la accidentabilidad mediante el desarrollo del Plan Nacional de Seguridad Vial. Esta comisión promueve actuaciones en los ámbitos de la vigilancia y el control, el acondicionamiento y servicios, la normativa, los estudios y programación y la educación. En este último caso, potencia la formación de los alumnos de educación infantil, primaria y secundaria mediante el fortalecimiento de valores y actitudes.

De manera específica se promueve la elaboración de materiales didácticos, la utilización de parques infantiles de tráfico, la celebración de jornadas de educación vial, el establecimiento de una asignatura optativa en la ESO, etc.

En las Comunidades autónomas durante los últimos treinta años se ha registrado una creciente responsabilización por las administraciones correspondientes en los ámbitos de la salud y la educación para la salud. Tanto en la legislación sanitaria y laboral como en la educativa es posible detectar un destacado interés hacia la promoción de la salud. A veces, esta preocupación se refleja en les leyes de sanidad propias de cada Comunidad. Otras mediante la creación de instituciones específicas coordinadas con las de carácter nacional como en el ámbito de la circulación vial o del consumo de drogas.

Así, en Galicia se crea en 1986 el Plan Galicia sobre Drogas en relación con el correspondiente Plan Nacional. Y en esta misma línea, en 1985 se crea el Observatorio de Galicia sobre Drogas siguiendo las recomendaciones de Europa donde se había creado en 1983 el Observatorio Europeo contra las toxicomanías. Esta institución nace con el objetivo de proporcionar una visión completa del fenómeno en sus diversas perspectivas facilitando así la toma de decisiones.

Al implantarse con la LOGSE (1990) la enseñanza de los temas transversales en la enseñanza obligatoria, se elaboraron diversos materiales didácticos correspondientes a los distintos temas.

En 1992 se estableció un convenio de colaboración entre las administraciones de sanidad y educación para el desarrollo de la educación para la salud en los centros 
escolares, dando prioridad a temas como la alimentación y nutrición, la prevención de drogodependencias, la sexualidad, la higiene personal, prevención de accidentes y salud mental.

Desde 1991 se publicó un material didáctico de apoyo al profesor, se trata de la guía "A experiencia de educar para a saúde na escola" Y desde 1993 se publicó la revista "Saudiña" que difundió en centros escolares interesantes experiencias relativas a los contenidos transversales y específicamente sobre la salud.

En 1995 la Consellería de educación dio a conocer en Galicia y promovió la Red Europea de Escuelas Promotoras de Salud

\section{Comunidad local}

Constituye el entorno social próximo a la persona, en el que se han a hacer operativas intervenciones que tienen su origen a nivel estatal y autonómico así como las que surjan de la propia comunidad. Los ayuntamientos pueden desempeñar un destacado papel en el diagnóstico de necesidades de la población, en la coordinación y control de actividades, en la solicitud de servicios, en la colaboración con otras administraciones y en su caso, en la creación y desarrollo de programas específicos.

En la actualidad y dentro de sociedades de carácter democrático se considera imprescindible la colaboración de los ciudadanos en todas las posibles fases de prestación de servicios sanitarios y educativos así como en otras actividades que tengan lugar en la comunidad a que pertenecen.

Uno de los ámbitos de intervención prioritaria de los ayuntamientos es el de la salud. En este sentido, parece necesario que existan actividades de prevención primaria, en las que promuevan estilos de vida saludables (actividades artísticas, deportivas, culturales...). Asimismo, se ha de dar una prevención de carácter secundario, mediante la existencia de servicios que faciliten el diagnóstico temprano y el tratamiento adaptado de problemas de salud. Finalmente, la comunidad ha de asumir también responsabilidades a nivel de atención terciaria relativos a la reinserción social de personas enfermas y el apoyo a sus familias.

Dentro de los ayuntamientos actúan los Centros de Salud, creados a partir de la estructuración de la atención sanitaria realizada en la Ley General de Sanidad de 1986. En ella se contempla una Atención Sanitaria Primaria, que correrá a cargo de los Centros de Salud y una atención Sanitaria Especializada que se realizará en los hospitales.

Los Centros de Salud se distribuyen según zonas territoriales y atienden a contingentes de población entre veinte y veinticinco mil personas. Entre sus principales objetivos se encuentran los de prevención, promoción y educación para la salud, que se han de proyectar en las personas, las familias y las instituciones de la comunidad. 
La función educadora de los ayuntamientos estrechamente vinculada con la promotora de salud ya se contemplaba en el informe "Aprender a ser" de la UNESCO en 1977 y con el paso del tiempo se ha fortalecido. Así, en 1990 tuvo lugar el I Congreso Internacional de Ciudades Educadoras y en 1994 el III Congreso, en el que se crea la Asociación Internacional de Ciudades Educadoras que elaboraría la Carta correspondiente, en la que se manifiesta el deber de los municipios de ejercer esta función contextualizándola dentro de una aspiración general de mejora de la calidad de vida.

En nuestro país existe un claro fundamento legal para la intervención de las comunidades locales en la educación de las personas. Así, las leyes del Ministerio de Educación como la LODE (Ley Orgánica del Derecho a la Educación, de 1985), la LOGSE, de 1990 o la LOE, del 2006.

Por otra parte, la Ley Reguladora de Bases de Régimen Local (LRBRL, de 1985), reconoce también de forma clara la capacidad de los ayuntamientos para participar en el funcionamiento de los servicios educativos. $Y$ de manera específica el Decreto 2774/1993 establece los cauces de cooperación entre el Ministerio de Educación y las corporaciones locales. En este sentido, los ayuntamientos colaboran con el sistema formal de enseñanza dependiente del ministerio o del gobierno autonómico mediante actuaciones como la cesión de terrenos para la construcción de centros, el mantenimiento de éstos una vez en funcionamiento, la participación en la admisión y en el control de asistencia de los alumnos, la facilitación de realización de actividades extraescolares y complementarias, etc. Por otra parte, los ayuntamientos pueden proceder también a la creación de centros y al desarrollo de programas educativos no suficientemente contemplados en las enseñanzas de régimen general como por ejemplo, las escuelas infantiles para niños de hasta tres años y las escuelas para personas adultas.

En la realización de programas específicos de educación para la salud a nivel de comunidad local se habrán de seguir una serie de fases como:

- El análisis de la realidad, que permita conocer cuáles son las características socioculturales, demográficas y sanitarias de la población a que se destina, especialmente, sus principales necesidades en salud y educación. Esta primera fase servirá para la fundamentación/justificación del programa.

- Determinación de los objetivos a conseguir, que se han de proyectar en los ámbitos de la prevención y la curación, así como en el aprendizaje de conocimientos, habilidades y actitudes.

- Selección y organización de contenidos o temas de trabajo, que se adaptarán a las necesidades de la población según temas de relevante actualidad sanitaria y de especial incidencia en determinadas edades o sectores de la comunidad. 
- Utilización de una metodología activa y motivadora, entendida como organización secuencializada de actividades, en las que deberá darse la participación de los destinatarios del programa.

- Utilización de recursos adecuados, entendidos en sentido amplio como personas, instalaciones y medios materiales así como de manera específica, medios didácticos (impresos, audiovisuales, informáticos, manipulativos...), que a veces podrán adoptarse de otros programas y otras deberán ser elaborados expresamente.

- La evaluación del programa se proyectará sobre todas las fases y componentes del mismo. Será por lo tanto una evaluación diagnóstica inicial, formativa o de procesos y de balance o final. Y abarcará no solo a alumnos sino también a profesores, recursos, metodología, etc.

Hay que destacar finalmente, que además de la intervención en colaboración con otras administraciones como las de sanidad y educación y de los programas y actividades que por si mismos puedan promover los ayuntamientos, estos pueden actuar como facilitadores de iniciativas privadas surgidas de forma voluntaria y con finalidades no lucrativas, como las correspondientes a ciertas ONGS y a asociaciones muy diversas como las de vecinos.

Se puede hacer referencia en este sentido al interés de algunas asociaciones de voluntarios por la promoción de actividades de carácter cultural, deportivo, artístico o medioambiental. También a la actuación del voluntariado en el acompañamiento y apoyo a personas con distintos niveles de dependencia. Resulta a veces de notable valor social la tarea de organizaciones de este tipo en la reinserción de enfermos de SIDA o drogodependientes a través de diversas iniciativas como los pisos de acogida.

También las asociaciones de vecinos se destacan a veces en tareas de promoción de la salud y la educación como por ejemplo, mediante el mantenimiento de escuelas vecinales.

\section{A modo de conclusión: Manifestaciones de la colaboración en educación para la salud}

Un análisis, por muy sencillo que se realice de la educación para la salud, pone de relieve la necesidad de una múltiple colaboración entre todos los agentes que en ella participan a fin de llevarla a término con éxito. A lo largo de las páginas anteriores se han puesto de relieve diversas formas de colaboración. Entre las múltiples posibilidades al respecto, parecen especialmente relevantes algunas como las siguientes: 
a) Entre los miembros de la familia y especialmente entre los padres a fin de presentar modelos coherentes de actuación ante multitud de situaciones que puedan inducir en sus hijos estilos de vida saludables.

b) Entre padres y profesores, dado que es conveniente la existencia de continuidad entre aprendizajes que se realizan en el hogar y aquellos que se fomentan en el colegio. Hay que advertir que no siempre existe un clima de entendimiento. La colaboración entre centro escolar y familias repercute positivamente en el aprendizaje del alumno, en la reducción del absentismo y de los problemas de disciplina y se pone de relieve en estos momentos con especial intensidad cuando la escolarización de los niños se realiza a edades cada vez más tempranas.

c) Entre profesores. La educación para la salud como el resto de los contenidos transversales presenta en la actualidad un tratamiento multidisciplinar, a través de las diversas áreas curriculares. Parece conveniente que se evolucione hacia un desarrollo interdisciplinar en el que se produzca una necesaria colaboración entre profesores a la hora de planificar, intervenir y evaluar las actividades correspondientes, de modo que se evite una enseñanza fragmentada dejando paso a otra más coherente y adaptada al alumno. Esta interdisciplinaridad solo será posible en la medida en que se de una estrecha colaboración entre los profesores de cada ciclo o departamento.

d) Entre profesores y especialistas. La tarea educadora se perfila de forma progresiva como un trabajo en equipo en el que es necesaria la coordinación de esfuerzos. La relación del profesor con especialistas en psicopedagogía, en pedagogía terapéutica, en medicina y en sociología parece especialmente recomendable en la enseñanza de los temas transversales y específicamente en educación para la salud, en la que ciertos temas como el consumo de drogas, la formación sexual o el SIDA pueden no ser bien dominados en ocasiones por el profesor.

e) Entre el colegio y el ayuntamiento, dadas las claras responsabilidades que tiene esta institución en el ámbito educativo. Como se ha indicado, se extienden a aspectos como el mantenimiento de los centros o el apoyo a la realización de actividades extraescolares y complementarias. Pero existe además otra forma de colaboración más global cuando el ayuntamiento, convirtiéndose en comunidad educadora promueve un entorno general estimulante de carácter formativo a través de la realización de actividades, de la apertura de sus instituciones al centro escolar, de la colaboración de sus funcionarios con el mismo...

f) Entre colegios y asociaciones de voluntarios. Diversas organizaciones de este tipo elaboran y ponen a disposición de los colegios programas de formación en temas de salud y material didáctico adaptado a su utilización en diversos niveles de enseñanza. A veces los alumnos se trasladan a las 
sedes de las ONGS y otras son miembros de estas organizaciones los que se trasladan al centro escolar para impartir charlas, seminarios o talleres sobre cuestiones de salud.

g) Entre administraciones. Como se ha indicado, existen actuaciones administrativas a nivel nacional, autonómico y local. Las competencias de los tres niveles administrativos deben estar estrechamente coordinadas, complementándose y reforzando su incidencia positiva sobre la educación para la salud.

h) Entre los medios de comunicación social, los colegios y las familias, dado que el niño y el joven permanecen una cantidad considerable de tiempo ante las pantallas. Es necesario que en la franja horaria de mayor audiencia por ellos, se emitan programas con valores formativos, reduciendo contenidos en publicidad y violencia.

\section{Referencias bibliográficas}

Ajuntament de Barcelona (1991). Primer Congreso Internacional de Ciudades Educadoras. Documentos finales. Barcelona: Ajuntament.

Costa, M. y López, E. (2000). Educación para la salud. Madrid: Pirámide.

Consellería de Sanidad (2003). Observatorio de Galicia sobre Drogas. Santiago: Xunta.

Consellería de Sanidad (2003). Plan de Salud de Galicia. Santiago: Xunta

Decreto 2774/1993 (22-XII) sobre cooperación de corporaciones locales con el M.E.C.

Galton, M. y Blyth, A. (1989).Handboock of Primary Education in Europe. Ed. Fulton. London: Council of Europe. Strasbourg

García Matilla, A. (2003). Una televisión para la educación. La utopía posible. Barcelona: Gedisa.

Federación Española de Municipios y Provincias (1987). La participación ciudadana en el municipio. Madrid: FEMP

Font, N. y Subirats, J. (2000). Municipios y sostenibilidad. Barcelona: Icaría.

García, A. et al. (2000). Educación para la salud: La apuesta por la calidad de vida. Madrid: Arán.

Gómez, J. R. (1991). Plan autonómico sobre drogodependencias de Galicia, principales líneas de actuación. Santiago: Dirección Xeral de Servicios Sociais. Xunta.

Ley 7/1985 de 2 de abril, Reguladora de las Bases de Régimen Local. BOE n. 80, de 3 de abril. 
Ley 14/1986 de 26 de abril, General de Sanidad. (B.O.E. n. 102, 29-IV-1986)

Ley 3/1986, do 18 de decembro de Consellos Escolares de Galicia. DOGA na 251 de 26 de decembro.

LOGSE: Ley Orgánica 1/1990 de 3 de octubre, de Ordenación General del Sistema Educativo. B.O.E. n. 238 de 4 de octubre.

Ley 7/2003 de 9 de diciembre de Ordenación Sanitaria de Galicia. B.O.E. n. 12 de 14 de enero

Ley 28/2005 de 26 de diciembre de medidas sanitarias frente al tabaquismo y reguladora de la venta, el suministro, el consumo y la publicidad de los productos del tabaco. (B.O.E. n. 309 de 27/12/2005)

LOE (Ley Orgánica de Educación. BOE n.106 del 4-V-2006)

Ley 42/2010 de 30 de diciembre (BOE n. 318 de 31 de diciembre de 2010) por la que se modifica la Ley $28 / 2005$ sobre medidas sanitarias frente al tabaquismo

M.E.C. (1993). Temas transversales y desarrollo curricular. Madrid: Publicaciones del MEC.

O.M.S. (1983). Nuevos métodos de educación sanitaria en la atención primaria de salud, en Informes técnicos, n. 690. Ginebra: OMS.

O.M.S. (1984). Educación para la salud. Manual sobre educación sanitaria en atención primaria de salud. Ginebra.

O.M.S. (2004). Informe sobre la salud en el mundo (SIDA). Ginebra.

Perea, R. (Direct. 2004). Educación para la salud: Reto de nuestro tiempo. Madrid: Díaz de Santos.

Piédrola Gil et al. (2003). Medicina preventiva y salud pública. Barcelona: Masson

Pino, M. (2001). La educación para la salud: marco teórico y desarrollo curricular. Vigo: Tórculo.

Plan Nacional Sobre Drogas (1992). Informe de situación y memoria de actividades. Madrid: Ministerio de Sanidad y Consumo.

Rosales, C. (2006). Perspectivas de educación para la salud. Santiago: Tórculo.

Subirats, J. (1999). ¿Existe sociedad civil en España? Responsabilidades colectivas y valores públicos. Madrid: Fundación Encuentro.

Subirats y otros (1999). La gestión y la organización de la administración educativa. Barcelona: UAB

Vega, A. (1993). La acción social ante las drogas. Propuestas de acción sociocomunitaria. Madrid: Narcea. 


\section{Nota biográfica}

Carlos Rosales López es Catedrático de Didáctica y Organizació Escolar en la Universidad de Santiago de Compostela. Sus líneas de investigación se centran en torno a la planificación de la enseñanza, la comunicación didáctica, la evaluación y los temas transversales. Email: carlos.rosales@usc.es 